\title{
GAIT ASYMMETRY DURING DUAL-TASK OBSTACLE CROSSING IN THE YOUNG AND ELDERLY
}

\author{
EMMANUEL S. DA ROCHA ${ }^{1}$, ÁLVARO S. MACHADO ${ }^{1}$, PEDRO S. FRANCO ${ }^{1}$, \\ ELIANE C. GUADAGNIN ${ }^{2}$, FELIPE P. CARPES ${ }^{1,2}$ * \\ ${ }^{1}$ Applied Neuromechanics Group, Laboratory of Neuromechanics, Federal University of Pampa, Uruguaiana, Brazil \\ ${ }^{2}$ Laboratory of Biomechanics, Federal University of Santa Maria, Santa Maria, Brazil
}

\begin{abstract}
Purpose. To evaluate gait asymmetry during obstacle crossing by young and elderly adults performing normal and dual-task gait. Methods. Ten healthy young adults and ten elderly adults with mild cognitive impairment performed a gait protocol by stepping over a foam obstacle during normal gait and while performing a secondary task (Stroop task). Sagittal kinematics of the lead and trail limbs were analyzed. Statistical procedures involved analysis of variance and tests at a significance of 0.05 . Results. Many of the kinematic variables presented a main effect for group (young adults vs. elderly adults), where the elderly featured poorer gait performance. It was observed that gait velocity during obstacle crossing in normal and dual-task gait was similar between the preferred and non-preferred limbs in both the young and elderly. However, the elderly were slower during normal and dual-task gait. A main effect for the dual-task condition was observed. Kinematic asymmetries for obstacle crossing were more frequent in the elderly and especially during the dual-task condition. Conclusions. The results suggest that the elderly may require more compensatory adjustments after crossing an obstacle. The asymmetries observed among the elderly may contribute to higher risk of falling during perturbed gait.
\end{abstract}

Key words: obstacle negotiation, ageing, functional lateralization, walking, secondary task, perturbed gait

\section{Introduction}

The difficulties that arise when stepping over an obstacle have been used to study locomotion in the elderly [1]. Previous studies have suggested that obstacles encountered on a walking pathway may increase the risk for tripping or slipping in the elderly [2, 3], as they require the adaptation of new gait strategies [4], more precise swing control, and higher levels of inter-joint coordination [5]. Additionally, protocols that combine gait with a secondary task were used to address the relationship between cognitive performance and gait ability in a context more similar to that experienced in daily life. When submitted to a dual-task condition, the elderly presented poorer gait performance than younger subjects [1]. Additionally, the negative effects of a secondary task are more pronounced in the elderly with dementia [6], even in cases with mild cognitive impairment [7]. Assessing gait when stepping over obstacles in a dualtask situation has been suggested as a potential tool for screening fall risk in the elderly [8]. However, there is very little research addressing the presence of gait asymmetry when elderly engage in dual-task walking.

"Split-belt" experiments have suggested that gait asymmetries lead to more failures in obstacle avoidance and require more attention to be paid by the elderly [9]. The limitation in performing executive functions during

\footnotetext{
* Corresponding author.
}

walking may increase asymmetry, which could affect dynamic stability [10]. As the elderly require adequate time to adapt foot placement strategies in relation to the obstacle as to avoid contact, dual-task gait would have similar effects on time constraints and might put the elderly in greater risk of contacting the obstacle [2]. Moreover, the elderly with strength asymmetry have greater gait asymmetries and gait variability than those without strength asymmetry [11].

The aim of this study was to address the presence of gait asymmetry in the elderly during obstacle crossing in normal and dual-task gait conditions and compare them with a population of young adults. It was believed that kinematic asymmetries during obstacle crossing in dual-task conditions would be more frequently observed in the elderly. This may suggest that while asymmetry may be part of regular gait performance, its impact on the risk of falling might differ between young and elderly subjects.

\section{Material and methods}

\section{Subjects}

Ten adult university students (six males, four females), aged $24.1 \pm 3.6$ years with a height of $1.69 \pm 0.12 \mathrm{~m}$ and body mass of $68 \pm 14.5 \mathrm{~kg}$, and ten elderly community-dwelling volunteers (four males, six females), aged $74.4 \pm 5.2$ years with a height of $1.57 \pm 0.05 \mathrm{~m}$ and body mass of $66 \pm 9 \mathrm{~kg}$, participated in our study. Subjects 
with neurological diseases (e.g., Parkinson's or Huntington's disease or who had suffered a stroke), vestibular or visual problems (clinically detectable), or with lower limb prosthesis were excluded. All subjects signed a written informed consent form approved by the local ethics committee (IRB no. \#0102011).

\section{Neuropsychological and mobility assessment}

Cognitive status was assessed using the Mini-mental State Examination (MMSE) [12]. Mental status was assessed using the Geriatric Depression Scale [13]. The Instrumental Activities of Daily Living Scale (IADL) [14] was used to quantity the independence of the subjects. Leg preference was verified using the "Waterloo inventory" [15].

\section{Gait assessment}

Participants performed a gait protocol with obstacles that had been previously used in other studies [3]. They performed the task while wearing their habitual shoes (flat, rubber-soled walking shoes) and clothes at self-selected comfortable speed along a $6 \mathrm{~m}$ walkway that had a foam obstacle positioned at the halfway point (height $15 \mathrm{~cm}$, width $34 \mathrm{~cm}$, length $11 \mathrm{~cm}$ ). A familiarization trial with and without the obstacle was permitted. Two valid trials were considered for each condition. Movement was recorded in the sagittal plane with a digital video camera (S2000HD, FUJI, Japan) at a resolution of 12.2 megapixels using a 1/2.3 inch CCD sensor, with the video later analyzed at $60 \mathrm{~Hz}$. Spherical reflexive markers were placed as anatomical references at the hallux, the fifth metatarsal head, and calcaneous tuberosity on both feet. A motion analysis tool (SkillSpector ver. 1.2.4, Video4coach, Denmark) was used to digitalize and track the markers providing position data. The camera was calibrated using a calibration frame with known dimensions. Estimated tracking error was $4 \mathrm{~mm}$, therefore gait motion was filmed in both directions, with the right limb and the left limb alternating in front of the camera, in order to minimize the influence of recording error. Toe clearance was calculated as the vertical dis- tance between the toe marker and the obstacle when the hallux marker of the swing limb was just crossing above the obstacle [16]. A Butterworth filter with a low-pass cut-off frequency of $6 \mathrm{~Hz}$ was applied to filter the data. The kinematics parameters monitored were: pre-obstacle trail limb step length, pre-obstacle trail limb distance, lead limb toe clearance, post-obstacle lead limb distance, lead limb stride length, and trail limb toe clearance (Fig. 1). Step variables were normalized for each subject's height and average velocity was computed for each trial.

The participants were requested to step over the obstacle during normal gait and while performing a variation of the Stroop task. In the dual-task condition, subjects were asked to verbally reply "yes" when the examiner said "blue" and "no" when the examiner said "red". For any other color that was named, the participants had to repeat the color (for example, if the examiner said "yellow", subject repeated "yellow"). For a trial to be considered valid, the dual-task condition was to be performed without stopping. Two valid trials were considered for analysis.

\section{Statistical analysis}

Data normality was verified using the Shapiro-Wilk test. Statistical comparisons were performed using analysis of variance in a $2 \times 2 \times 2$ mixed model by analyzing group (elderly and adult), leg (preferred and non-preferred), and condition (normal and dual-task gait) with Bonferroni corrections for multiple comparisons. When main effects or interactions were observed, kinematic data were compared by applying paired $t$ tests. Non-parametric data were compared using the Wilcoxon test. The level of significance was set at 0.05 .

\section{Results}

\section{Neuropsychological and mobility}

The cognitive status of the young adults, assessed using the MMSE, was higher than 26 points indicating cognitively normal status. The elderly were found to
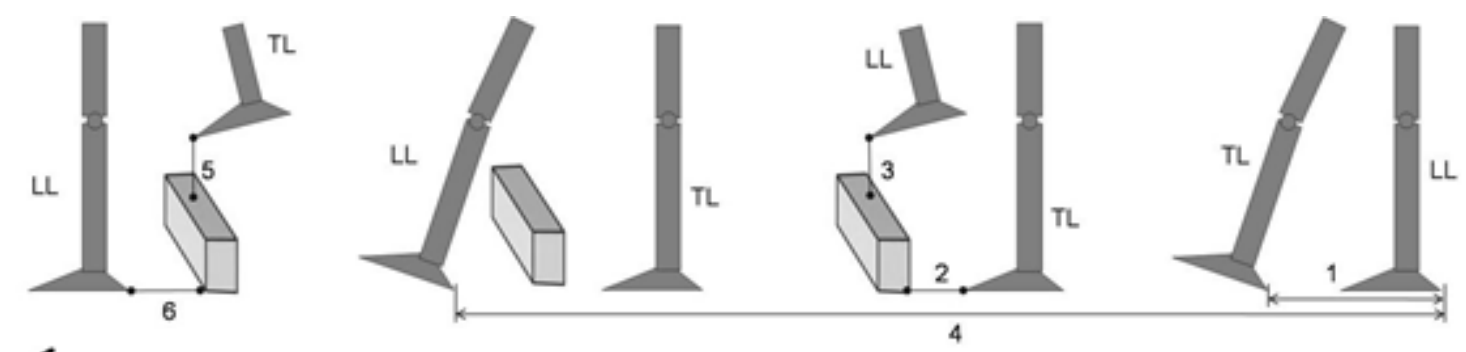

(1) pre-obstacle trail limb step length (4) lead limb stride length

(2) pre-obstacle trail limb distance (5) trail limb toe clearance

(3) lead limb toe clearance

(6) post-obstacle lead limb distance; black arrow denotes the movement direction

Figure 1. Lower limb movements during the obstacle crossing task, where LL represents the lead limb (the first limb to step over the obstacle) and TL the trail limb (the contralateral limb to step over the obstacle) 
E.S. da Rocha et al., Gait asymmetries during obstacle crossing

present mild cognitive impairment as based on MMSE scores between 20 and 26 points $[15,16]$. The results from the Geriatric Depression Scale excluded the risk of depression, and all subjects were classified as independent in regard to daily life tasks.

\section{Gait analysis}

When performing normal gait during obstacle crossing with the non-preferred limb, average gait velocity was $0.91 \pm 0.26 \mathrm{~m} / \mathrm{s}$ for the elderly and $1.11 \pm 0.13 \mathrm{~m} / \mathrm{s}$ for the young adults. When comparing the groups, it was observed that gait velocity when crossing the obstacle during normal $[Z=-0.105 ; p=0.917]$ and dual-task gait $[Z=-1.402 ; p=0.161]$ was similar between the preferred and non-preferred limbs among the young adults. The same was observed for the elderly in normal $[Z=-0.663 ; p=0.508]$ and dual-task gait $[Z=-1.126$; $p=0.260]$. During the dual-task condition while crossing an obstacle with the preferred limb, average velocity was $0.71 \pm 0.15 \mathrm{~m} / \mathrm{s}$ for the elderly and $0.97 \pm 0.16 \mathrm{~m} / \mathrm{s}$ for the young adults. When performing the dual-task condition while crossing an obstacle with the non-preferred limb, average gait velocity was $0.78 \pm 0.26 \mathrm{~m} / \mathrm{s}$ for the elderly and $1.04 \pm 0.16 \mathrm{~m} / \mathrm{s}$ for the young adults. The elderly were slower than the young adults during normal gait performance under obstacle-crossing conditions with the preferred $[Z=-2.016 ; p=0.044]$ and non-preferred limbs $[Z=-2.191 ; p=0.028]$. The same was observed for dual-task gait performance while crossing the obstacle with the preferred $[Z=-2.524 ; p=0.012]$ and non-preferred limbs $[Z=-2.193 ; p=0.028]$. Gait velocity was similar when crossing the obstacle with the preferred or non-preferred limb.

An effect for group in pre-obstacle trail limb step length, post-obstacle lead limb distance, and lead limb stride length was found, which were lower in the elderly (Fig. 2). An effect for leg was observed in pre-obstacle trail limb distance (greater in the preferred leg for both groups) and post-obstacle lead limb distance (lower in the preferred leg for both groups). An effect for condition was observed in both groups for pre-obstacle trail limb step length and pre-obstacle trail limb distance, which was greater in the dual-task condition. Significant interactions were observed between group and leg for trail limb toe clearance, group and condition interaction for post-obstacle lead limb distance, and leg and condition interaction for lead limb toe clearance. The corresponding $F$ and $p$ values are presented in Tables 1 and 2 .

While symmetry was observed in normal gait, the dual-task condition elicited greater pre-obstacle trail limb step length in the preferred leg in the elderly $\left[t_{(9)}\right.$ $=-4.212 ; p=0.002]$ and in the non-preferred leg in the young adults $\left[\mathrm{t}_{(9)}=-2.659 ; p=0.026\right.$ ].

Pre-obstacle trail limb distance was found to be asymmetric among the young adults during normal gait $\left[\mathrm{t}_{(9)}=3.22 ; p=0.01\right]$ and for the elderly in the dual-

Table 1. Statistical outcomes considering the effects of group, leg, and condition by analysis of variance ( $F$ and $p$ values)

\begin{tabular}{lrrrrrr}
\hline \multirow{2}{*}{ Variable } & \multicolumn{2}{c}{ Group } & \multicolumn{2}{c}{ Leg } & \multicolumn{2}{c}{ Condition } \\
\cline { 2 - 7 } & $F$ & $p$ & $F$ & $p$ & $F$ & $P$ \\
\hline Pre-obstacle trail limb step length & 6.45 & $0.032^{*}$ & 0.09 & 0.76 & 6.05 & $0.03^{*}$ \\
Pre-obstacle trail limb distance & 2.59 & 0.14 & 17.75 & $0.002^{*}$ & 5.85 & $0.03^{*}$ \\
Lead limb toe clearance & 1.81 & 0.21 & 0.79 & 0.39 & 3.10 & 0.11 \\
Post-obstacle lead limb distance & 47.14 & $>0.001^{*}$ & 10.24 & $0.011^{*}$ & 0.08 & 0.77 \\
Lead limb stride length & 9.68 & $0.01^{*}$ & 4.64 & 0.06 & 0.29 & 0.60 \\
Trail limb toe clearance & 3.76 & 0.08 & 1.95 & 0.19 & 0.21 & 0.65 \\
\hline
\end{tabular}

* statistically significant at $p<0.05$

Table 2. Statistical outcomes considering the interactions of group $\times$ leg; group $\times$ condition; leg $\times$ condition; and group $\times$ leg $\times$ condition ( $F$ and $p$ values)

\begin{tabular}{lcccccccc}
\hline \multirow{2}{*}{ Variable } & \multicolumn{2}{c}{ Group $\times$ leg } & Group $\times$ condition & Leg $\times$ condition & $\begin{array}{c}\text { Group } \times \text { leg } \\
\times \text { condition } \\
\end{array}$ \\
\cline { 2 - 9 } & $F$ & $p$ & $F$ & $p$ & $F$ & $p$ & $F$ & $p$ \\
\hline Pre-obstacle trail limb step length & 1.42 & 0.26 & 0.56 & 0.47 & 0.09 & 0.76 & 2.74 & 0.13 \\
Pre-obstacle trail limb distance & 0.07 & 0.79 & 0.002 & 0.96 & 0.41 & 0.53 & 3.44 & $0.09 *$ \\
Lead limb toe clearance & 0.003 & 0.96 & 3.12 & 0.11 & 14.31 & $0.004^{*}$ & 0.98 & 0.34 \\
Post-obstacle lead limb distance & 0.21 & 0.65 & 10.1 & $0.01^{*}$ & 0.001 & 0.97 & 0.06 & 0.80 \\
Lead limb stride length & 3.91 & 0.07 & 2.18 & 0.17 & 0.35 & 0.56 & 0.04 & 0.83 \\
Trail limb toe clearance & 15.73 & $0.003^{*}$ & 1.55 & 0.24 & 0.25 & 0.62 & 0.19 & 0.67 \\
\hline
\end{tabular}

* statistically significant at $p<0.05$ 

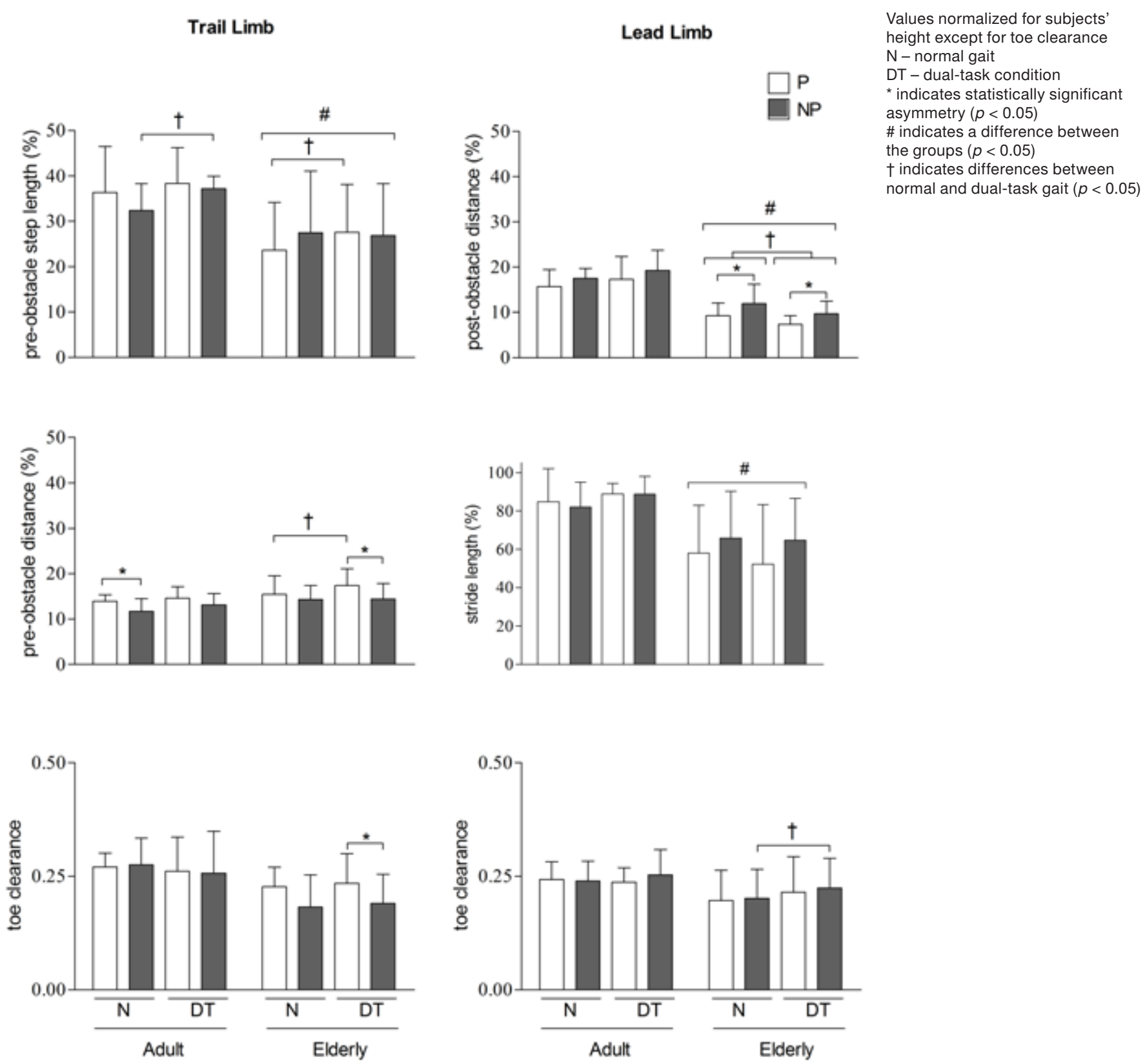

Figure 2. Kinematic data for lead and trail limbs, where the white bars represent data from the preferred leg (P) and black bars represent data from the non-preferred leg (NP)

task condition $\left[\mathrm{t}_{(9)}=3.42 ; p=0.008\right]$. For both groups, the values were greater in the preferred leg. In the dualtask condition, the elderly increased the pre-obstacle trail limb distance of the preferred leg $\left[\mathrm{t}_{(9)}=-3.174 ; p=0.011\right]$, although this was not observed in the young adults.

The elderly presented asymmetry in the post-obstacle lead limb distance in both normal $\left[\mathrm{t}_{(9)}=-4.26 ; p=0.002\right]$ and dual-task conditions $\left[\mathrm{t}_{(9)}=-3.51 ; p=0.007\right]$. Moreover, a condition effect was observed for this variable in the elderly group, with lower values in the dual-task condition than during normal gait for both the preferred $\left[\mathrm{t}_{(9)}=2.93 ; p=0.017\right]$ and non-preferred legs $\left[\mathrm{t}_{(9)}=3.47 ; p=0.007\right]$. Increased lead limb toe clearance was observed in the elderly performing the dual-task condition with the non-preferred limb when compared with the young adults $\left[\mathrm{t}_{(9)}=-2.803 ; p=0.021\right]$, but no asymmetry was detected.

The elderly in the dual-task condition presented asymmetry in trail limb toe clearance, which was greater with the preferred leg $\left[\mathrm{t}_{(9)}=2.39 ; p=0.04\right]$. Despite this asymmetry, during the dual-task condition there were no significant changes in the magnitude of trail limb toe clearance in the elderly during the normal and dual-tasking conditions (Fig. 2).

\section{Discussion}

Dual-task conditions are often observed in daily life, such as when walking while talking to other individuals. When crossing obstacles, the elderly are known to require 
E.S. da Rocha et al., Gait asymmetries during obstacle crossing

a longer period of time for stepping over an obstacle [17]. Previous studies on the elderly have suggested an increased risk of tripping or falling when attention is divided during walking in the presence of expected $[18,19]$ or unexpected obstacles [1]. However, little attention has been paid to the issue of asymmetry in locomotion. Here, the asymmetry in the kinematics of gait over obstacles during normal and dual-task conditions for young adults and the elderly were studied. In general, our data suggest that gait during obstacle-crossing situations in a dual-task condition elicits asymmetries primarily in the elderly. Additionally, the results on the elderly suggest that the non-preferred leg seems to be more affected in a dual-task condition than the preferred leg.

Lower extremity asymmetries may increase the risk of falling in the elderly, as had been suggested in a study about asymmetry in leg extension strength and power in individuals with and without a history of falls [20,21]. Additionally, lower extremity strength asymmetry was recently correlated with gait asymmetry and variability in the elderly, which was reinforced when they were performing near maximal capability [11].

The findings of our study demonstrate that the obstacle-approaching phase differs between the young and elderly. The elderly presented asymmetry in the trail limb kinematics with effects found for both groups and the dual-task condition. The differences, asymmetries, and effects of a dual-task condition in the studied groups suggest that the elderly may require more compensatory adjustment after crossing an obstacle, since the trail limb presented effects for both groups and the dual-task condition, with asymmetric toe clearance in the dualtask condition.

Based on these observations, it can be assumed that the elderly may prefer a more consistent gait pattern by shortening step and stride length [22]. However, this did not result in them producing similar movements for the preferred and non-preferred leg. The asymmetry presented by the elderly during obstacle crossing is consistent with the asymmetric propulsion pattern found in the muscle moments and power patterns of the elderly [23].

In the group of elderly, asymmetry was observed in the toe clearance of the trail limb but not in the lead limb. This suggests that the elderly may place greater attention on the first limb when crossing an obstacle, but feature impaired control of gait symmetry when the trail limb crosses. Asymmetry in foot clearance was observed among the elderly with a high risk of falling [10] and may reflect functional asymmetries that are needed for gait stability [24]. Indeed, most of studies on gait asymmetry in the elderly considered the measure of toe or foot clearance [25]. The influence of the dualtask condition on lead limb toe clearance may suggest that the elderly place more attention on the lead limb as it is the first leg to cross an obstacle, but then pay less attention during the second crossing with the trail limb, which was noted to feature significant asymmetry in the dual-task condition.

When considering the foot-ground clearance presented by older adults, a recent study reported asymmetries regardless of whether they performed treadmill and overground walking [25]. In both cases, minimum toe clearance was greater with the non-preferred foot. The authors suggested that the non-preferred limb works primarily in the stabilization of gait [25]. Our data on step and stride kinematics suggest that obstacle crossing may induce significant asymmetries in the elderly. Even considering this study's small sample size, this could generally indicate that gait performed over obstacles may rely on specific responses depending on which leg is first used by the elderly.

The protocol presented here suggests that a bilateral assessment of gait during obstacle crossing requires further attention. One of the limitations of this study was the use of a single camera for recording movement, although it may be useful for clinics and hospitals when addressing elderly mobility without the possibility of using several cameras simultaneously.

\section{Conclusions}

The results suggest that the elderly feature kinematic asymmetries during obstacle crossing especially in a dualtask condition. The group differences, asymmetries, and effects of dual-task gait suggest that the elderly may use additional compensatory adjustment after crossing an obstacle.

\section{Acknowledgments}

This study was funded by the Brazilian National Counsel of Technological and Scientific Development (CNPq) by grant No. 476163/2010-2. The authors would like to thank Dr. Jacques Duysens for his comments in preparing the manuscript.

\section{References}

1. Hegeman J., Weerdesteyn V., van den Bemt B., Nienhuis B., van Limbeek J., Duysens J., Dual-tasking interferes with obstacle avoidance reactions in healthy seniors. Gait Posture, 2012, 36 (2), 236-240, doi: 10.1016/j.gaitpost.2012.02.024.

2. Galna B., Peters A., Murphy A.T., Morris M.E., Obstacle crossing deficits in older adults: A systematic review. Gait Posture, 2009, 30 (3), 270-275, doi: 10.1016/j.gaitpost. 2009.05.022.

3. Chen H.L., Lu T.W., Wanga T.M., Huang S.C., Biomechanical strategies for successful obstacle crossing with the trailing limb in older adults with medial compartment knee osteoarthritis. J Biomech, 2008, 41 (4), 753-761, doi: 10.1016/j.jbiomech.2007.11.017.

4. Zhang C., Mao D., Riskowski J.L., Song Q., Strategies of stepping over obstacles: The effects of long-term exercise in older adults. Gait Posture, 2011, 34 (2), 191-196, doi: 10.1016/j.gaitpost.2011.04.008.

5. Wang T.M., Yen H.C., Lu T.W., Chen H.L., Chang C.F., Liu Y.H. et al., Bilateral knee osteoarthritis does not affect inter-joint coordination in older adults with gait 
deviations during obstacle-crossing. J Biomech, 2009, 42 (14), 2349-2356. doi: 10.1016/j.jbiomech.2009.06.029.

6. Beauchet O., Allali G., Berrut G., Hommet C., Dubost V., Assal F., Gait analysis in demented subjects: Interests and perspectives. Neuropsychiatr Dis Treat, 2008, 4 (1), 155-160.

7. Ijmker T., Lamoth C.J.C., Gait and cognition: The relationship between gait stability and variability with executive function in persons with and without dementia. Gait Posture, 2012, 35 (1), 126-130, doi: 10.1016/j.gaitpost.2011.08.022.

8. Beauchet O., Annweiler C., Dubost V., Allali G., Kressig R.W., Bridenbaugh S. et al., Stops walking when talking: a predictor of falls in older adults? Eur J Neurol, 2009, 16 (7), 786-795, doi: 10.1111/j.1468-1331.2009.02612.x.

9. McFadyen B.J., Hegeman J., Duysens J., Dual task effects for asymmetric stepping on a split-belt treadmill. Gait Posture, 2009, 30 (3), 340-344, doi: 10.1016/j.gaitpost. 2009.06.004.

10. Di Fabio R.P., Kurszewski W.M., Jorgenson E.E., Kunz R.C., Footlift asymmetry during obstacle avoidance in highrisk elderly. J Am Geriatr Soc, 2004, 52 (12), 2088-2093, doi: 10.1111/j.1532-5415.2004.52569.x.

11. Laroche D.P., Cook S.B., Mackala K., Strength asymmetry increases gait Asymmetry and variability in older women. Med Sci Sports Exerc, 2012, 44 (11), 2172-2181, doi: 10.1249/MSS.0b013e31825e1d31.

12. Folstein M.F., Folstein S.E., McHugh P.R., "Mini-mental state". A practical method for grading the cognitive state of patients for the clinician.J Psychiatr Res, 1975, 12 (3), 189-198, doi: 10.1016/0022-3956(75)90026-6.

13. Yesavage J.A., Brink T.L., Rose T.L., Lum O., Huang V., Adey M. et al., Development and validation of a geriatric depression screening scale: a preliminary report. J Psychiatr Res, 1982, 17 (1), 37-49, doi: 10.1016/0022-3956 (82)90033-4.

14. Lawton M.P., Brody E.M., Assessment of older people: self-maintaining and instrumental activities of daily living. Gerontologist, 1969, 9 (3 Part 1), 179-186, doi: 10. 1093/geront/9.3_Part_1.179.

15. Elias L.J., Bryden M.P., Bulman-Fleming M.B., Footedness is a better predictor than is handedness of emotional lateralization. Neuropsychologia, 1998, 36 (1), 37-43, doi: 10.1016/S0028-3932(97)00107-3.

16. Liu M.W., Hsu W.C., Lu T.W., Chen H.L., Liu H.C., Patients with type II diabetes mellitus display reduced toe-obstacle clearance with altered gait patterns during obstaclecrossing. Gait Posture, 2010, 31, 93-99, doi: 10.1016/j. gaitpost.2009.09.005.

17. Uchiyama M., Demura, S., Sugiura, H. The mobility performance of the elderly before, during and after cross- ing over an obstacle. Hum Mov, 2012, 13 (4), 297-302, doi: 10.2478/v10038-012-0034-1.

18. Muir S.W., Speechley M., Wells J., Borrie M., Gopaul K., Montero-Odasso M., Gait assessment in mild cognitive impairment and Alzheimer's disease: The effect of dualtask challenges across the cognitive spectrum. Gait Posture, 2012, 35 (1), 96-100, doi: 10.1016/j.gaitpost.2011.08.014.

19. Harley C., Wilkie R.M., Wann J.P., Stepping over obstacles: Attention demands and aging. Gait Posture, 2009, 29 (3), 428-432, doi: 10.1016/j.gaitpost.2008.10.063.

20. Perry M.C., Carville S.F., Smith I.C., Rutherford O.M., Newham D.J., Strength, power output and symmetry of leg muscles: effect of age and history of falling. Eur J Appl Physiol, 2007, 100 (5), 553-561, doi: 10.1007/s00421006-0247-0.

21. Skelton D.A., Kennedy J., Rutherford O.M., Explosive power and asymmetry in leg muscle function in frequent fallers and non-fallers aged over 65. Age Ageing, 2002, 31 (2), 119-125, doi: 10.1093/ageing/31.2.119.

22. Chen H.C., Ashton-Miller J.A., Alexander N.B., Schultz A.B., Stepping over obstacles: gait patterns of healthy young and old adults. J Gerontol, 1991, 46 (6), M196-203, doi: 10.1093/geronj/46.6.M196.

23. Sadeghi H., Prince F., Zabjek K.F., Labelle H., Simultaneous, bilateral, and three-dimensional gait analysis of elderly people without impairments. Am J Phys Med Rehabil, 2004,83(2),112-123, doi:10.1097/01.PHM.0000107484. 41639.2C

24. Nagano H., Begg R.K., Sparrow W.A., Taylor S., Ageing and limb dominance effects on foot-ground clearance during treadmill and overground walking. Clin Biomech (Bristol, Avon), 2011, 26 (9), 962-968, doi: 10.1016/j. clinbiomech.2011.05.013.

25. Barrett R.S., Mills P.M., Begg R.K., A systematic review of the effect of ageing and falls history on minimum foot clearance characteristics during level walking. Gait Posture, 2010, 32 (4), 429-435, doi: 10.1016/j.gaitpost.2010. 07.010.

Paper received by the Editors: October 2, 2012

Paper accepted for publication: April 16, 2013

\section{Correspondence address}

Felipe P. Carpes

Federal University of Pampa

Laboratory of Neuromechanics

BR 472 km 592

Po box 118 - ZIP 97500-970

Uruguaiana, RS, Brazil

e-mail: carpes@unipampa.edu.br 